\title{
The effect of short-term consumption of vitamin D Supplements following a session of increasing activity on respiratory parameters and toleratance to activity of women with mild asthma
}

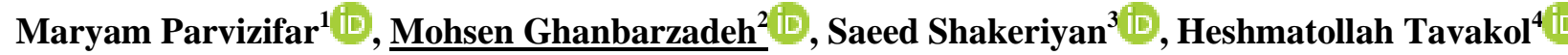 \\ ${ }^{1}$ Department of Exercise Physiology, Faculty of Sport Sciences, Shahid Chamran University of Ahvaz, Ahvaz, Iran \\ ${ }^{2}$ Corresponding author; Department of Exercise Physiology, Faculty of Sport Sciences, Shahid Chamran University of Ahvaz, \\ Ahvaz, Iran \\ Email:m.parvizifar@gmail.com \\ Department of Exercise Physiology, Faculty of Sport Sciences, Shahid Chamran University of Ahvaz, Ahvaz, Iran \\ ${ }^{4}$ Department of Internal Medicine, School of Medicine, Ahvaz, Jundishapur University of Medical Sciences, Iran
}

Citation Parvizifar M, Ghanbarzadeh M, Shakeriyan S, Tavakol H. [ The effect of short-term consumption of
vitamin D Supplements following a session of increasing activity on respiratory parameters and toleratance to
activity of women with mild asthma]. J Birjand Univ Med Sci. 2020; 27(1): 80-9. [Persian]

\begin{abstract}
Background and Aim: Exercises to reduce respiratory symptoms of asthma and Vitamin D by regulating the immune system can play a significant role in the health of asthmatic patients. The purpose of the present study was to investigate the effect of short-term consumption of vitamin D supplements following a session of increasing activity on respiratory parameters and tolerance to activity of women with mild asthma.

Materials and Methods: This study was quasi-experimental based on the pre-test and post-test pattern that following the announcement of the call, 30 asthmatic women volunteered in Ahwaz was performed. Ballk test for cardiopulmonary patients was used for increasing aerobic exercise. Supplementation with vitamin D tablets at a dose of 1000 units daily was performed for six weeks. For evaluation of pulmonary Vital Capacity (VC) and Maximum Voluntary Ventilation (MVV) indices, the Spirometer device was used. To determine normality of the data was used Shapiro-Wilk's test and data analysis by using paired t-test.
\end{abstract}

Results: The results showed that after the intervention, there was a significant difference in VC and MVV levels and tolerance to activity in the pre-test, supplementation and exercise stages $(\mathrm{P} \leq 0.05)$.

Conclusion: Taking vitamin D supplementation with aerobic exercise can improve respiratory function in asthmatic patients and increase tolerance to activity.

Key Words: Aerobic Exercise Increasing; Asthma; Maximal Voluntary Ventilation; Vitamin D Supplementation; Vital Capacity 


\section{تأثير مصرف كوتاهملت مكمل ويتامين D متعاقب يك جلسه فعاليت فز آينله بر يارامتر هاى تنفسى و تحمل به فعاليت زنان مبتلا به آسم خفيف}

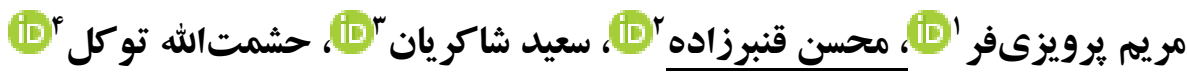

جكيده

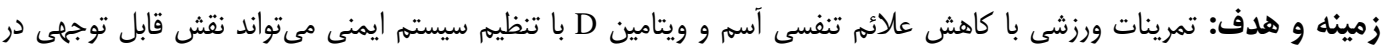

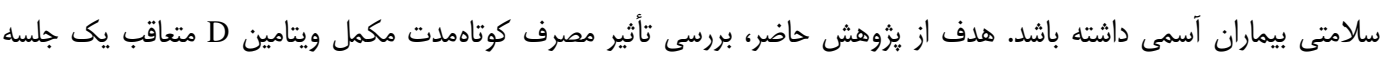

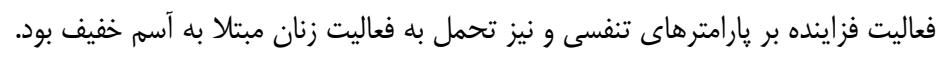

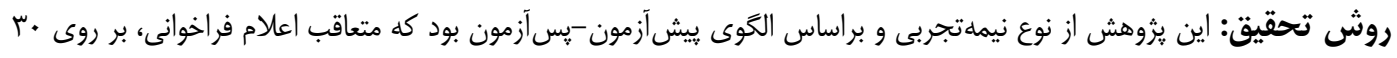

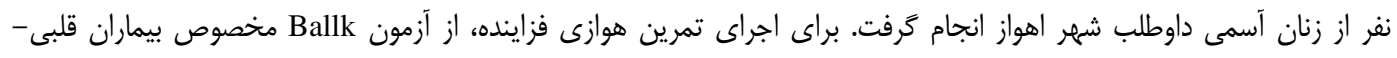

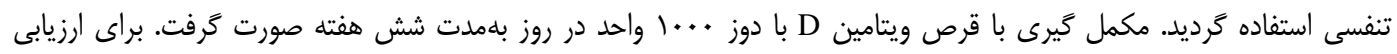

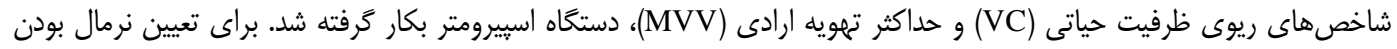

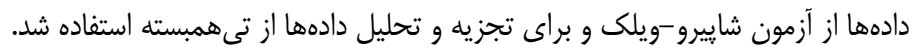

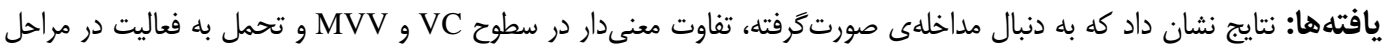

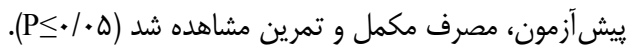
نتيجهَّيرى: مصرف مكمل ويتامين D همراه با ورزش هوازى مىتواند موجب بهبود عملكرد دستخاه تنفسى در بيماران آسمى شود و تحمل به فعاليت را افز ايش دهد. وازههاى كليدى: تمرين هوازى فزاينده؛ آسم؛ حداكثر تهويه ارادى؛ مكمليارى ويتامين D؛ ظرفيت حياتى

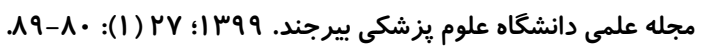

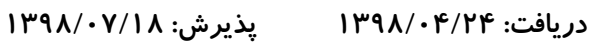

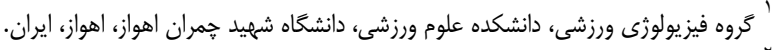

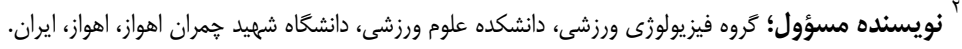

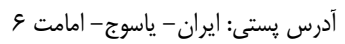
m.parvizifar@gmail.com بست الكترونيكى تلفن:

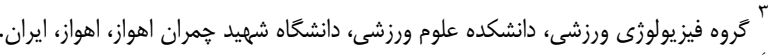

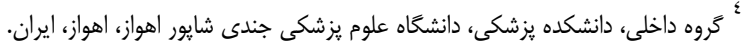


بهدنبال تمرينات هوازى، تغييرات و سازكارىهاى ساختارى مقدمه

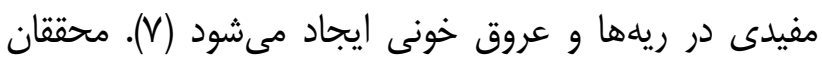

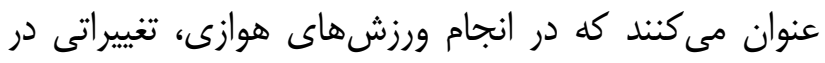
سيستم عضلانى، قلبى - عروقى و ريوى اتفاق مئى افتند كه

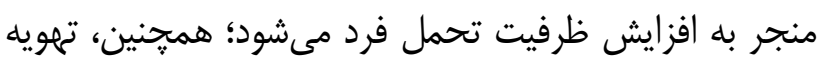

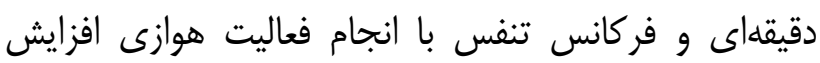

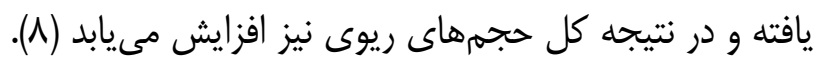

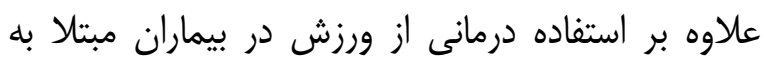

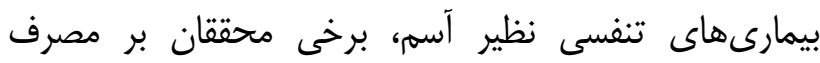

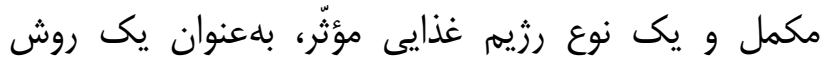

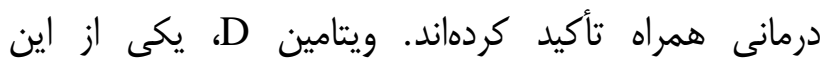

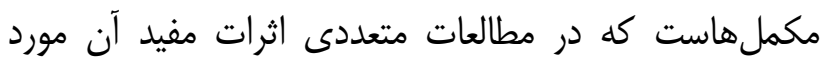

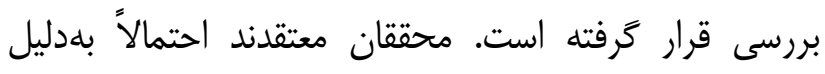

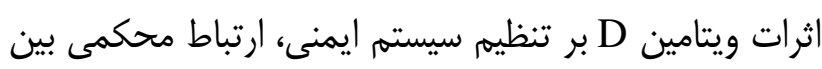

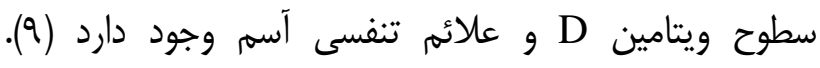

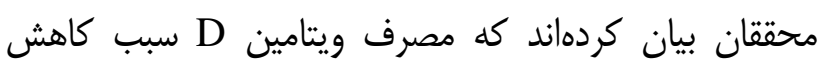

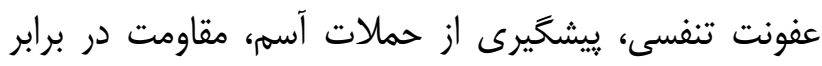

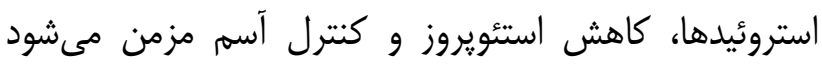
(•) (1). ويتامين D اثرات مفيدى در مسيرهاى ضدّ التهابى ريه

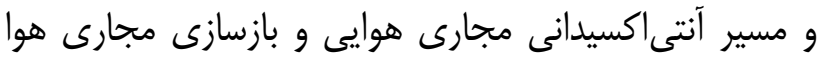

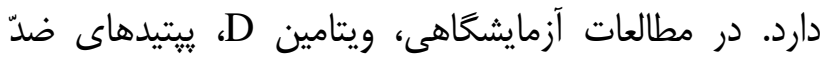

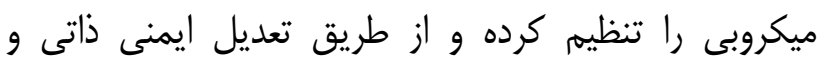

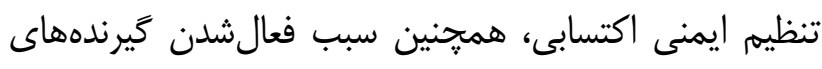

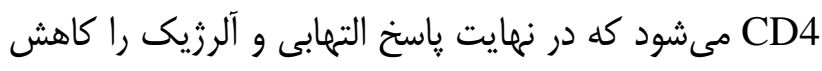

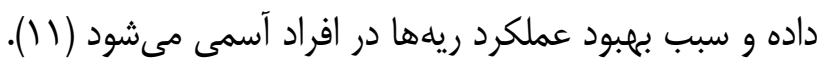

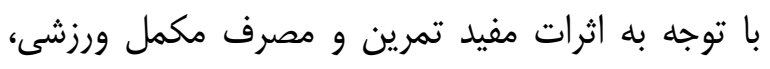

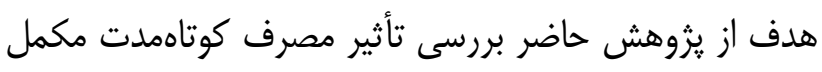

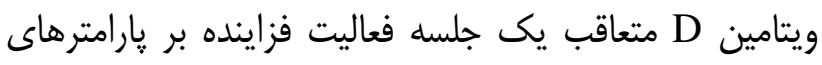
تنفسى و تحمل به فعاليت زنان مبتلا به آسم خفيف بودي بودي

بيمارىهاى مزمن ريوى، از شايعترين بيمارىهايى

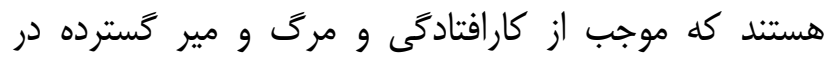
جوامع بشرى مىشوند. در اين ميان، يكى شئ شايعترين

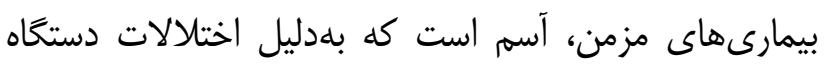

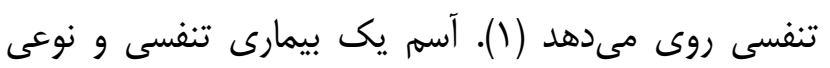

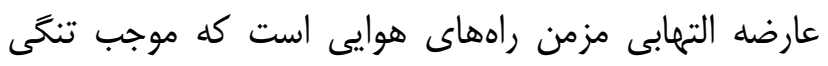

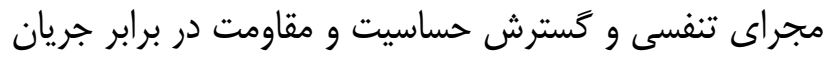

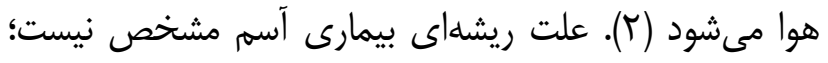

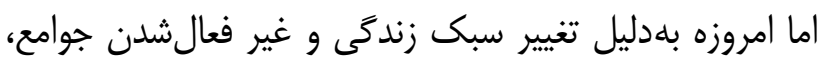

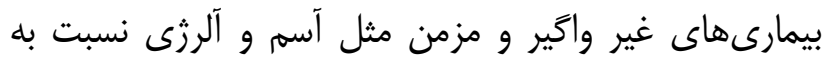

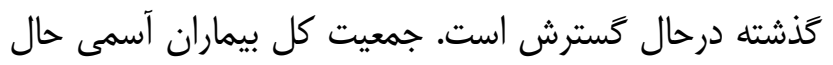

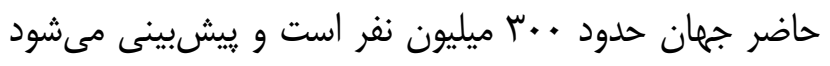

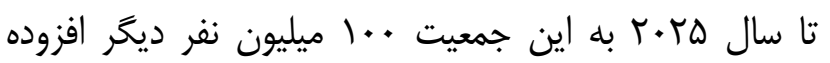
شود (ז).

شواهد يزوهشى، از تأثير مطلوب تمرينات هوازى بر

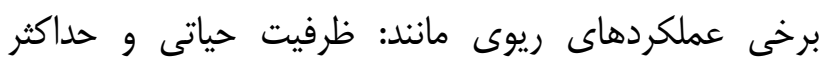

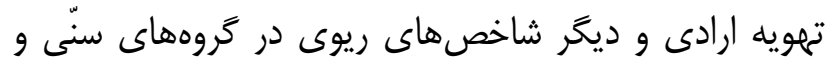

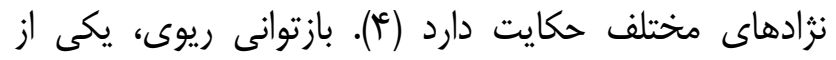
روشهاى شناختهشده براى بهبود علائم بيمارى آسم و ورئ

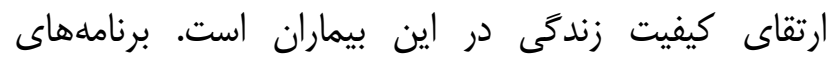

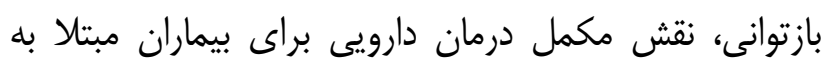

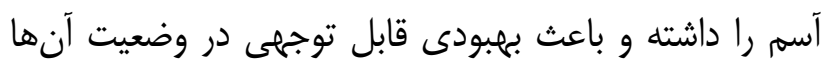

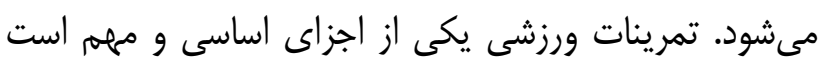

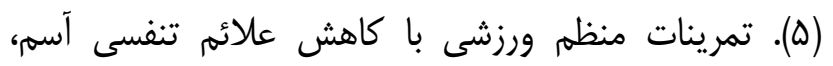

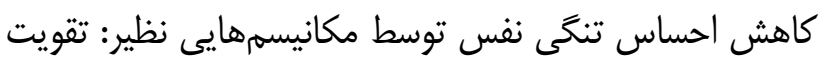

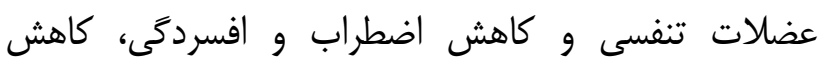

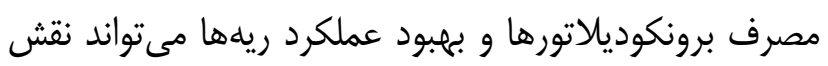

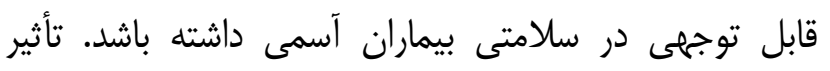
فعاليتهاى هوازى بلعنوان يكى از تمرينات ورزشى رايج، بر بران

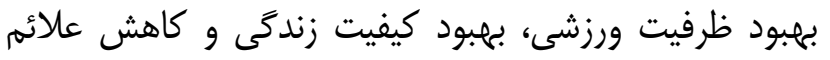

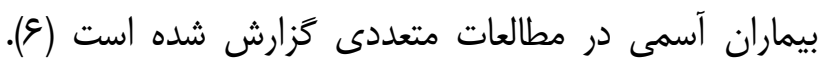




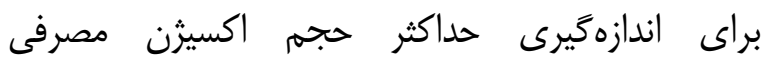
روش تحقيق

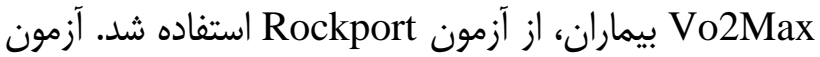
Rockport

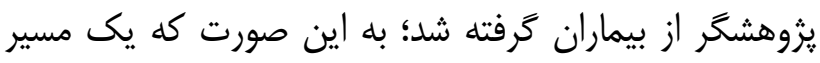

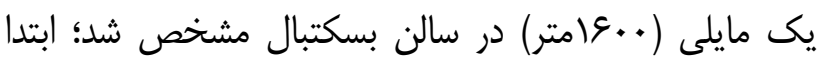

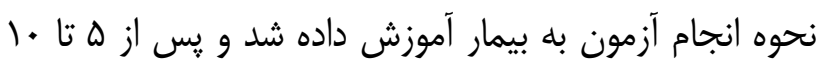

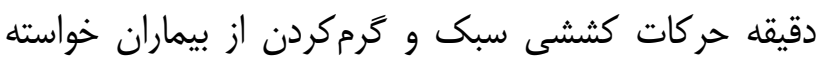

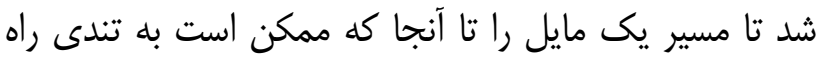

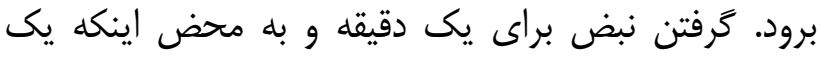

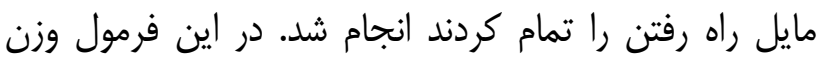
بلن بر حسب يوند، سن بر حسب سال، فاكتور جنسيت با ران

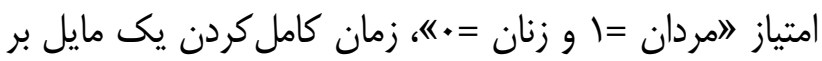
حسب دقيقه و ضربان قلب پِ از انجام اين تست بر حسب دان "ضربه بر دقيقه است كه در فرمول زير وارد مىشود (T) (I).

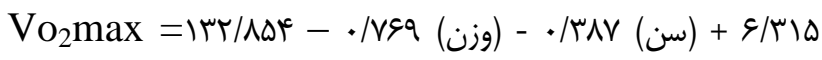

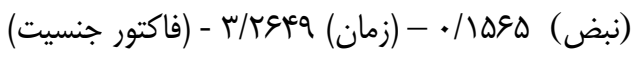

ابتدا مشخصات افراد مورد مطالعه از قبيل: سن، قد، وزن و اطلاعات محيطى از قبيل دما و رطوبت نسبى آزمايشگاه و ارتفاع محل از سطح دريا براى كاليبرهكردن دستخاه اسبيرومترى ديجيتال (مدل IF8 ساخت كشور آلمان) وارد

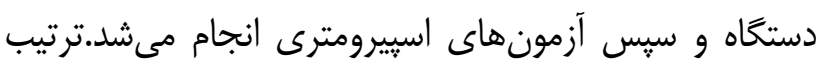

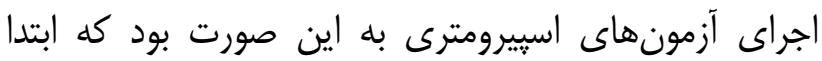

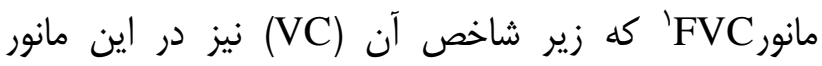

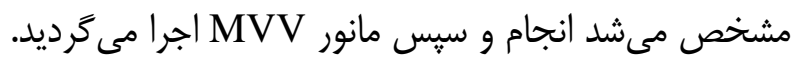
آزمون FVC: براى اجراى آزمون VC مىبايست ابتدا

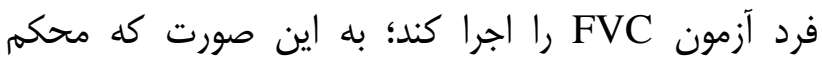

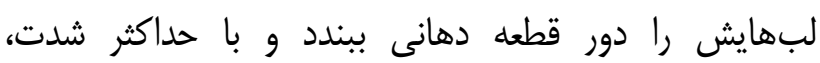

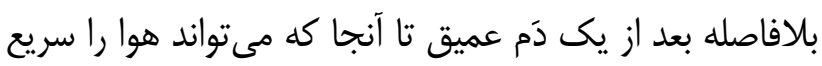
و با شدت فوت كند؛ ببهطورى كه بيشترين حجم ريه در ثانيه

مطالعه حاضر از نوع نيمهتجربى و كاربردى بود. جامعه آمارى در اين مطالعه شامل تمام زنان آسمى شهرستان اهواز

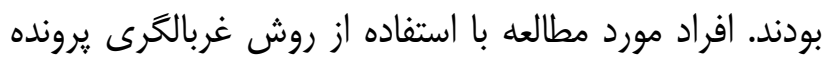

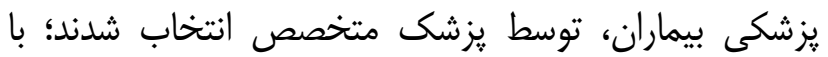

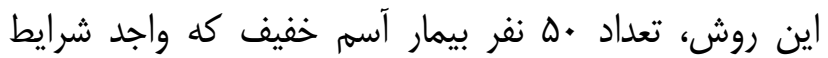

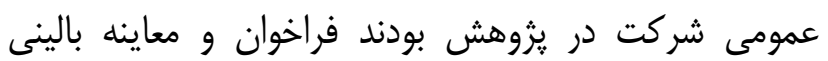

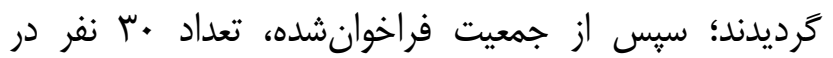
دسترس و علاقمند به شركت در طرح كه انجام فعاليتهاى

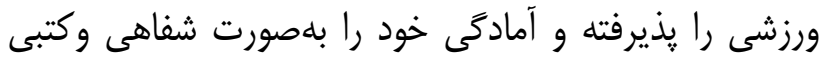
اعلام نمودند، انتخاب شدند.

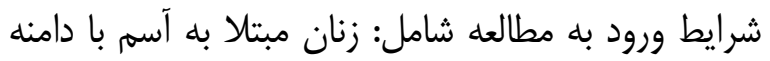

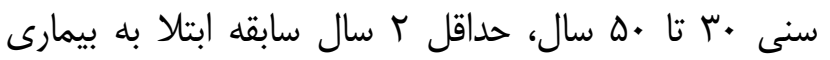

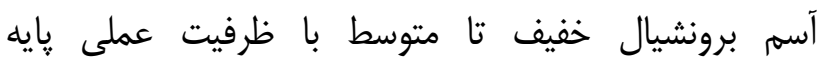

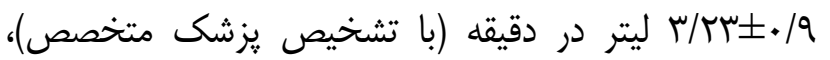

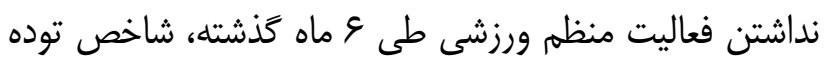

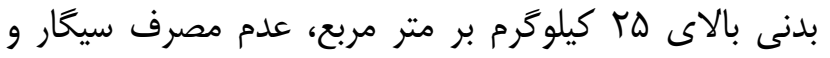

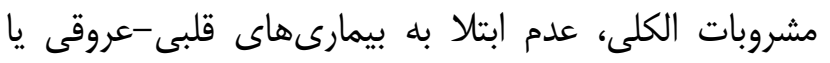

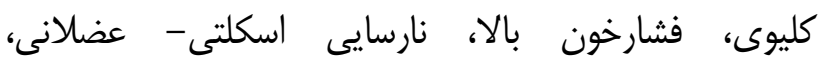
سبرىشدن حداقل س ماه از آخرين عود بيمارى، داشتن درجه

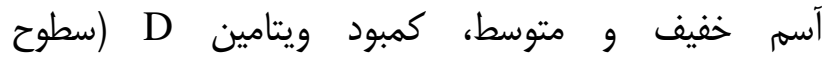

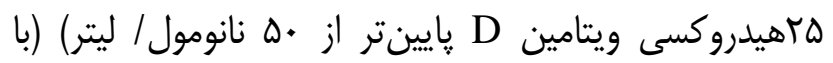
تشخيص يزشك متخصص) بودند. يس از توضيح اهداف و روش انجام فعاليتهاى بدنى، از

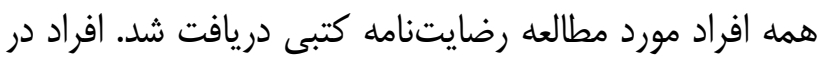

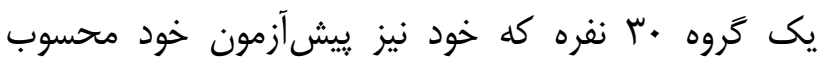

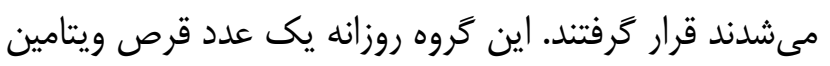

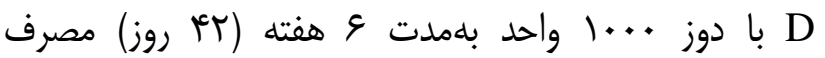

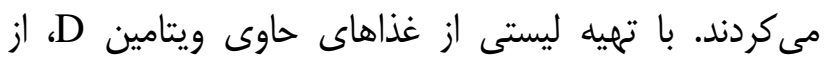
بيماران خواسته شد اين غذاها را مصرف نكنند.

\footnotetext{
${ }^{1}$ Forced Vital Capacity
}

ثبت دادههاى يزووهث: 
شيب تا زمان خستخى و واماندگى اضافه مىشد. سرعت تا

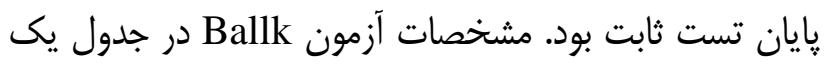
نشان داده شده است.

\section{روشهاى آمارى:}

در يزوهش حاضر نرمالبودن دادهها با استفاده از آزمون

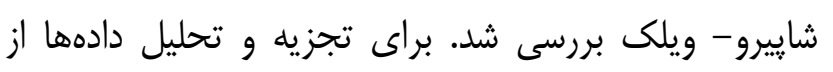

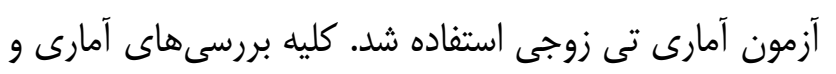

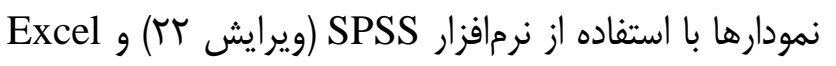

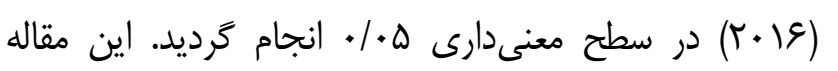
داراى Ir.medilam.rec.1396.0240377881

جallk جدول ا - مراحل آزمون

\begin{tabular}{|c|c|c|c|}
\hline مرحله & زمان (دقيقه ) & شيب (درصد ) & سرعت (كيلومتر / ساعت) \\
\hline 1 & $\cdot-1$ & . & $\Delta / \kappa^{c}$ \\
\hline r & $1-4$ & r & $\Delta / T^{c}$ \\
\hline r & $r-\mu$ & r & $\Delta / \uparrow^{c}$ \\
\hline f & $r-r$ & f & $\Delta / \uparrow^{c}$ \\
\hline$\Delta$ & $f-\phi$ & $\Delta$ & $\Delta / \Gamma^{c}$ \\
\hline 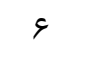 & $\Delta-\Phi$ & 9 & $\Delta / \Gamma^{c}$ \\
\hline v & $q-\gamma$ & v & $\Delta / \Gamma^{c}$ \\
\hline$\wedge$ & $\vee-\Lambda$ & $\wedge$ & $\Delta / \Gamma^{c}$ \\
\hline 9 & $1-9$ & 9 & $\Delta / \varsigma^{c}$ \\
\hline 1. & $9-1$. & 1. & $\Delta / \uparrow^{c}$ \\
\hline 11 & $1 \cdot-11$ & 11 & $\Delta / \uparrow^{\circ}$ \\
\hline IT & $11-14$ & ir & $\Delta /{ }^{c}$ \\
\hline سו & سו-rו & سו & $\Delta / \Gamma^{c}$ \\
\hline 15 & $\mid r-1 f$ & if & $\Delta / \kappa^{c}$ \\
\hline 10 & $15-10$ & 10 & $\Delta / \kappa^{c}$ \\
\hline
\end{tabular}

مشخصات فردى افراد مورد مطالعه در جدول ب آورده شده است. با توجه به جدول سا، نتايج آزمون t همبسته بيانگر آن است كه سطوح MC و MVV سرمى بيماران مورد بررسى در مراحلِ ييشآزمون و مرحله مصرف مكمل، تغييرات

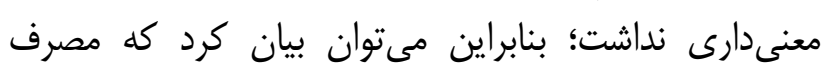

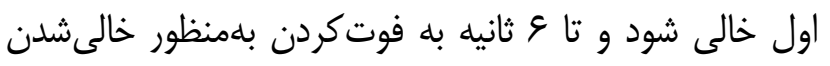

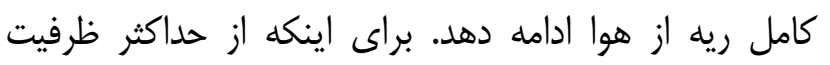

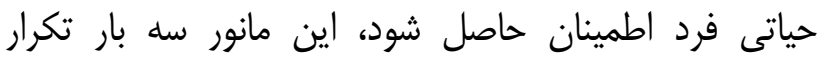

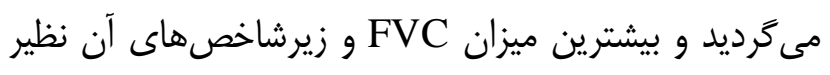
بازدم سريع در ثانيه اول (FEV1) (FEF) (PEF)

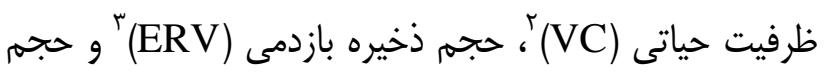

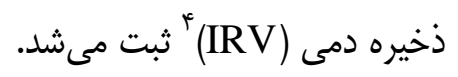
آزمون حداكثر تهويه ارادى (MVV)

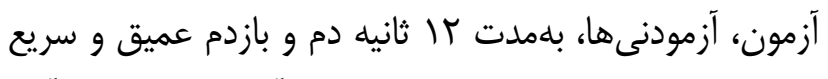

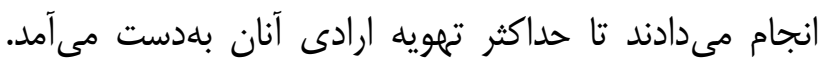

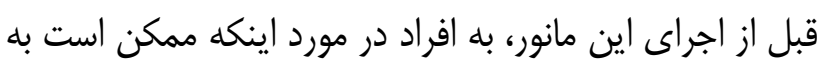

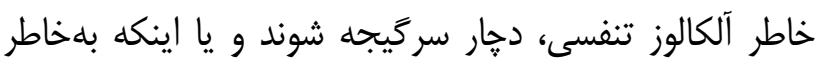

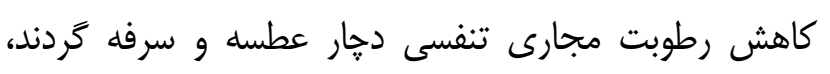

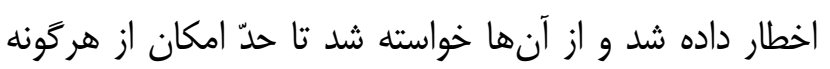

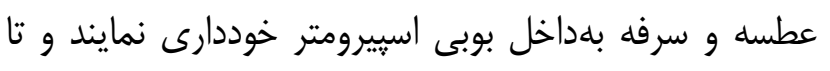
اتمام r إنانيه، به تنفس سريع خود با همان ريتهم يكنواخت

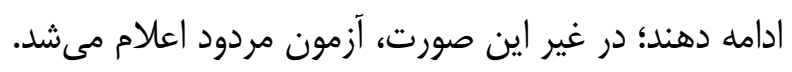

\section{برنامه ورزشى:}

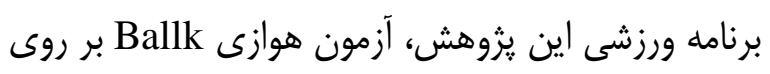
تردميل بود كه طى دو مرحله تكجلسه (قبل از شروع مرحله

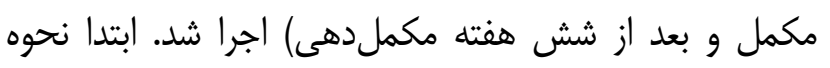

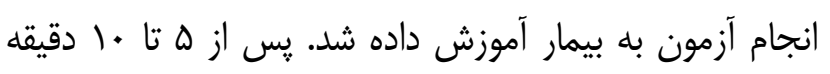

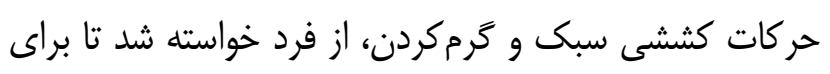

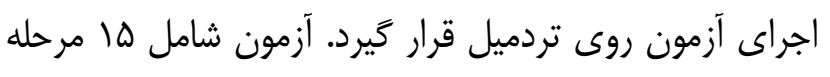

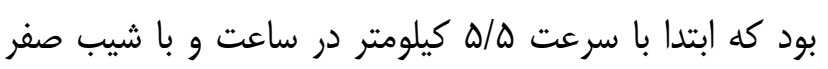

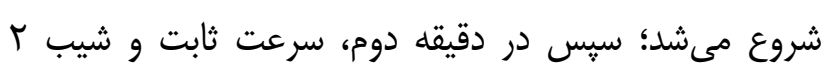

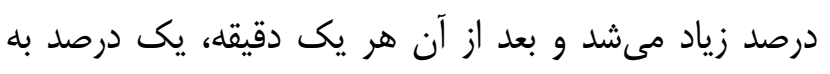

\footnotetext{
${ }^{1}$ Forced Expiratory Volume in First Second

${ }^{2}$ Vital Capacity

${ }^{3}$ Expiratory Reserve Volume

${ }^{4}$ Inspiratory Reserve Volume

${ }^{5}$ Maximal Voluntary Ventilation
} 


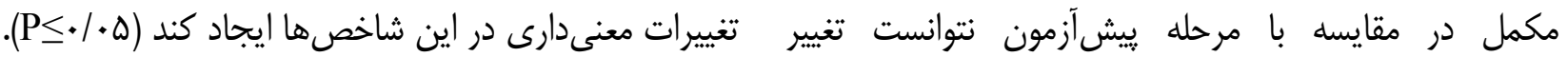

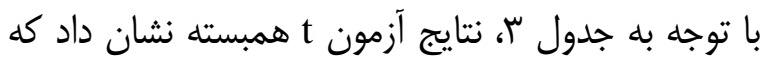
معنى دارى در اين شاخص ايجاد كند (ه) (P> (P)؛ اما سطوح

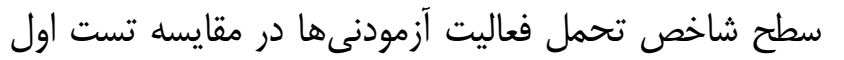
Ballk سرمى افراد مورد مطالعه در مراحل تمرين و و

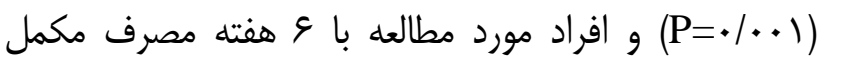
مرحله مصرف مكمل - تمرين تغييرات معنىدارى داشته است است مورد مقايسه با مرحله فقط انجام فعاليت هوازى فزاينده توانست توان فوانستند مدتزمان زيادى در در تست Ballk فعاليت كنند.

جدول r - تركيب بدن و مشخصات فردى افراد مورد مطالعه

\begin{tabular}{|c|c|c|c|c|c|}
\hline (كيلوَّرم بر مجذور توده بدن & (ميلى ليتر / كيلوكَرم/ / دقيقنه مصرفى & (درصد) & (كيلوترم) & (سانتى متر) & (سال) \\
\hline$r F / \Delta \cdot \pm 1 / T r$ & $\Gamma \Delta / \cdot . \pm \Gamma / \wedge \Delta$ & $r \Delta / \cdot \pm \pm r / / q$ & $s \mathrm{~V} / \Delta \mathrm{V} \pm \mu / \cdot \mathrm{V}$ & $1 q V / \Delta \cdot \pm q /<q$ & $\Gamma / \mathcal{M} \pm \Gamma / \Delta V$ \\
\hline
\end{tabular}

جدول س- نتايج آزمون آمارى t زوجى شاخص هاى MVV،VC و تحمل فعاليت بين دو مرحله يِش آزمون و پِ آزمون

\begin{tabular}{|c|c|c|c|}
\hline \multirow[b]{2}{*}{ سطوح معنىدارى } & \multicolumn{2}{|c|}{ مراحل آزمون } & \multirow[b]{2}{*}{ شاخص } \\
\hline & 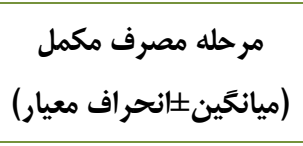 & (ميانغين土|نحراف آزمون معيار) & \\
\hline.$/ . \wedge \varepsilon$ & $r / . . \pm \cdot / \% q$ & 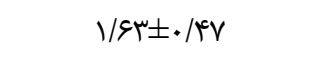 & (ليتر) VC \\
\hline \multirow[t]{2}{*}{.$/ 494$} & $F N / F F \pm F / F \varphi$ & 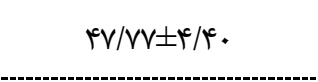 & MVV \\
\hline & \multicolumn{2}{|c|}{ مراحل آزمون } & \\
\hline سطوح معنىدارى & (ميانكَين دانحر مكمل - تمرين معيار) & 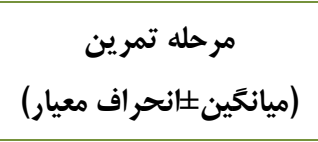 & شاخص \\
\hline$\% v V$ & $r / M \pm \cdot / Q T$ & $r / r \Lambda \pm \cdot / \Lambda \Lambda$ & (ليتر) VC \\
\hline \multirow[t]{2}{*}{$\%$} & 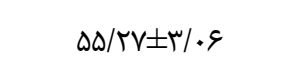 & $\Delta \cdot / \cdot \pm \Delta / 19$ & MVV \\
\hline & \multicolumn{2}{|c|}{ مراحل آزمون } & \multirow[b]{2}{*}{ شاخص } \\
\hline سطوح معنىدارى & 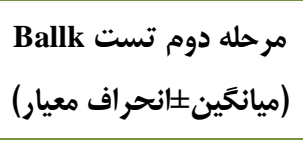 & 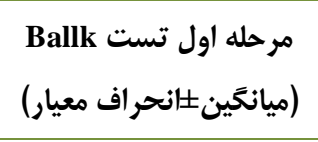 & \\
\hline $.1 . .1$ & $11 / V V \pm 1 / \Delta F$ & ( & تحمل فعاليت (دقيقه) \\
\hline
\end{tabular}

VC: vital capacity

MVV: Maximum Ventilatory Volume 
(1) (1). يزوهشهاى خسروى و همكاران نشان داد كه V هفته

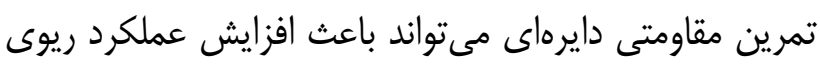

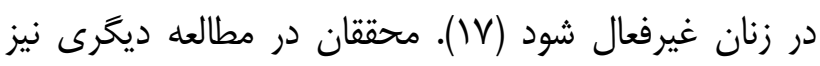

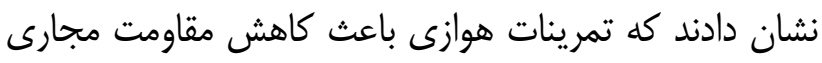

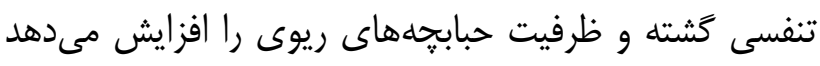

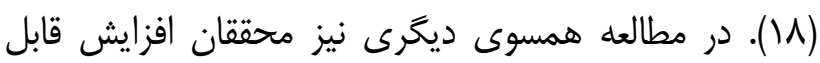

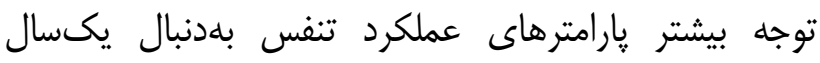

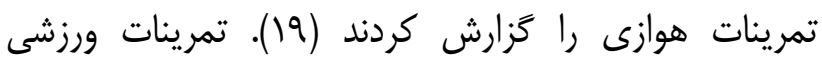

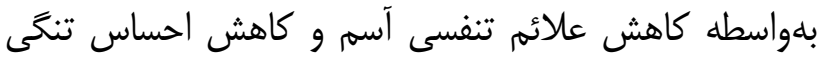

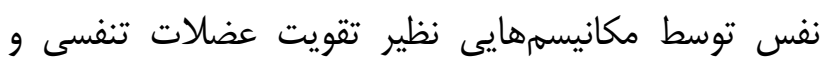
كاهش مصرف داروهاى متعدد براى بهبود بيمارى آسم و

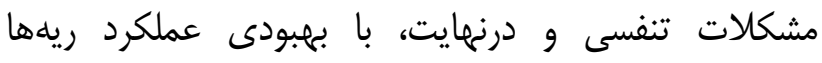
مى تواند نقش مهمى در بهبود بيمارى مبتلايان به آسم داشته

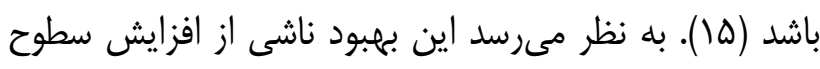

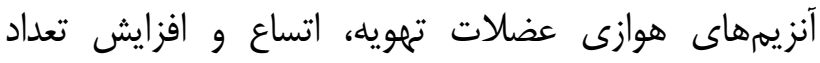

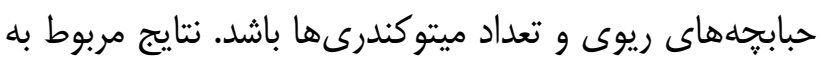
مطالعه حاضر را نيز مىتوان به اين مكانيسمها مران مرتبط دانست؛ زيرا در يثوهش حاضر نيز افراد مورد مطالعه با انجام تمرينات

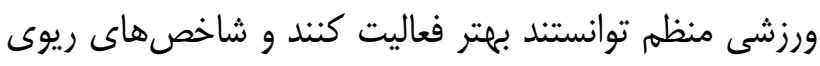

آنها نيز بلهورت معنى دارى بهبود داشت.

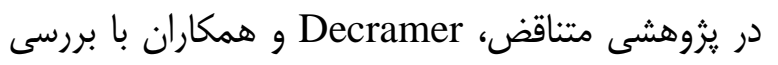

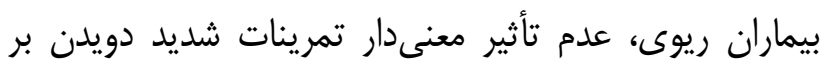

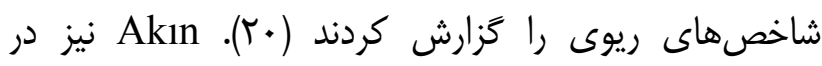

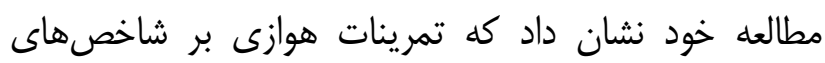
سرعت اوج بازدمى، حجم بازدمى با فشار، حجم بازدم با فشار

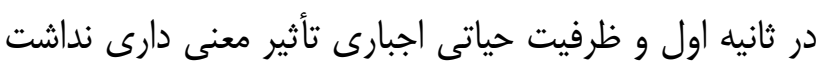

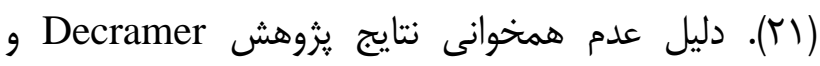

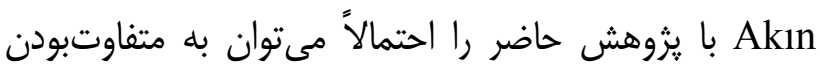
دوز ويتامين D، متفاوتبودن نوع آزمودنىها و شرايط آزمون

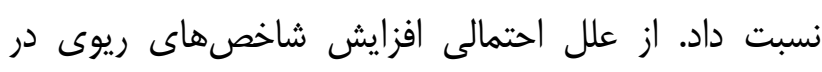
يثزوش حاضر، مىتوان به وجود ويتامين D نيز اشاره نمود.

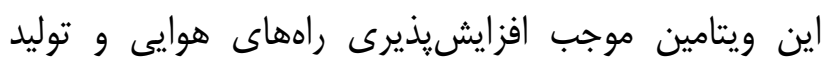

مهمترين يافته اين يزوهش، حاكى از اين بود كه سطوح

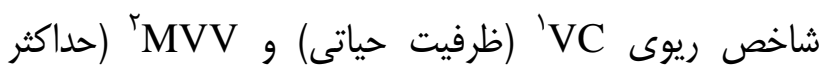
تهويه ارادى)، آزمودنىها در مرحله تمرين هوازى همراه با رياتى

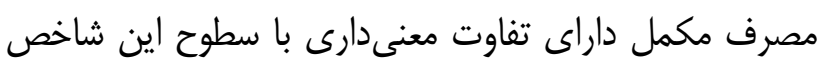

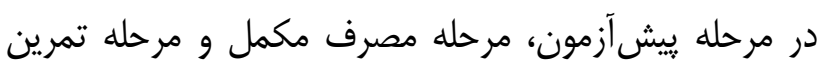
بود. محققان در مطالعات متعددى به اثرات مفيد مصرف مكمل D بر بهبود شاخصهاى ريوى اشاره كردهاند. در مطالعهاى، ارتباط بين سطح ويتامين D، شدت آسم و عملكرد

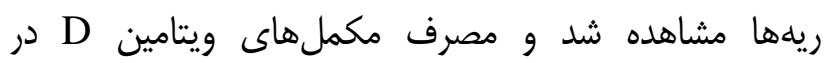

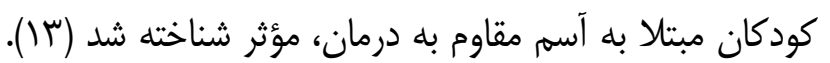
همجنين در مطالعه ديخرى مشخص شده است است كه كه كمبود

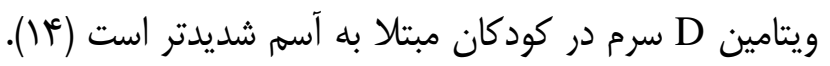

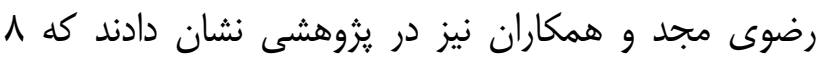

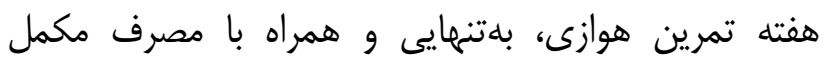

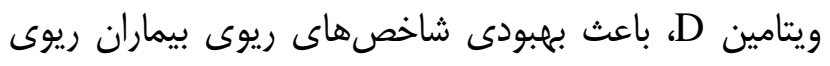

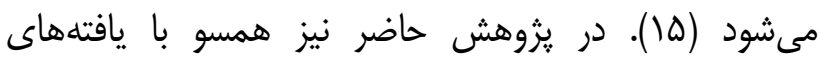

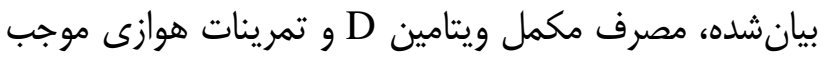

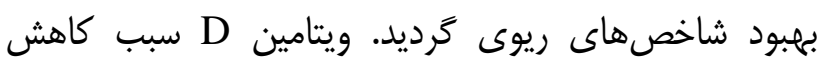

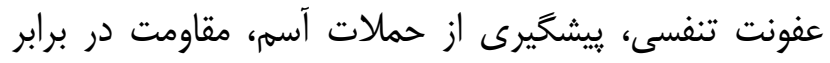

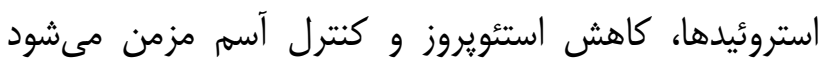

فعاليتهاى جسمانى بلهور گسترداى در بيماران مبتلا

به بيمارىهاى ريوى با هدف توانبخشى، مورد استفاده قرار مى

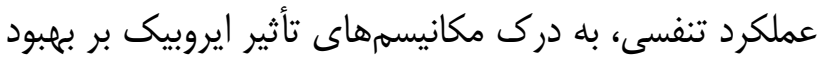

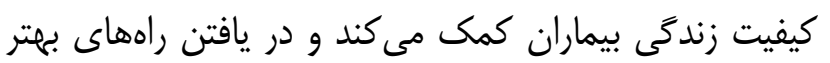
براى توانبخشى نيز مفيد است؛ با اين وجود، مطالعات بسيار كمى در رابطه با ورزش هوازى و عملكرد ريه ويه وجود دارد

\footnotetext{
${ }^{1}$ Vital capacity

${ }^{2}$ Maximal Voluntary Ventilation
} 
شاخصهاى عملكرد ريوى و ميزان عملكرد هوازى بيماران

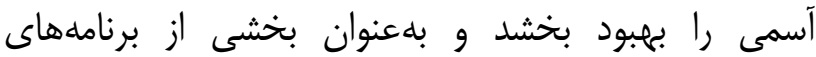
توانبخشى ريوى بيماران آسمى، مدّ نظر قرار گيرد (^).

\section{نتيجه كيرى}

در كل، نتايج بيانگر اين است كه انجام فعاليت هوازى از طريق تقويت عضلات تنفسى و ساير عوامل فيزيولوزيكى

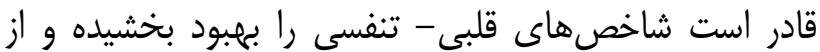

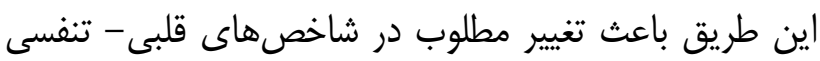

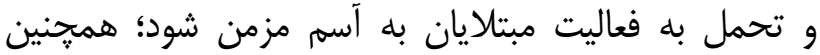

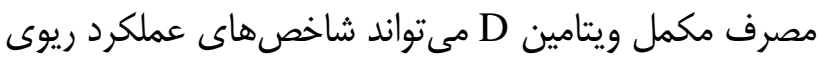

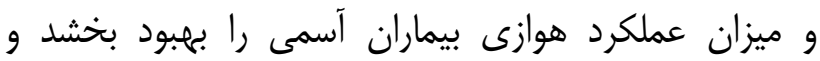

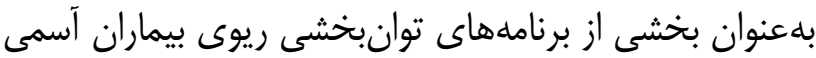

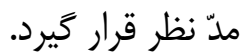

\section{تقدير و تشكر}

اين مقاله برگرفته از يايان نامه كارشناسى ارشد رشته تربيت بدنى، گرايش فيزيولوزى ورزشى مصوب در درئ دانشگاه

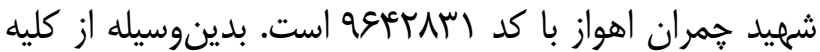

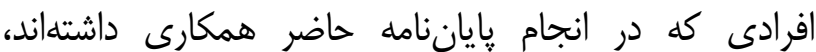
صميمانه قدردانى و تشكر مىشود.

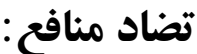

نويسندكان مقاله اعلام مى دارند كه هيج گَونه تضاد منافعى در يزوهش حاضر وجود ندارد.
يبتيدهاى ضد ميكروبى و از طرف ديگر، ورزش موجب افزايش ترشح هورمون خاراتيروئيد مىشود؛ لازم به يه يادآورى است كه كليهها از نقاطى هستند كه تحت تأثير تأثير اين هورمون

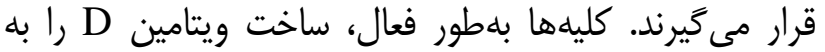

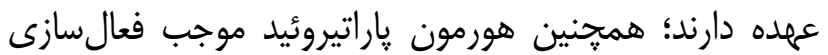
ويتامين D و اين ويتامين خود باعث توليد ايتترلوكين-

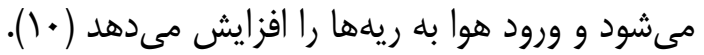
در يزوهش حاضر، بيماران با انجام فعاليت همراه با رهان

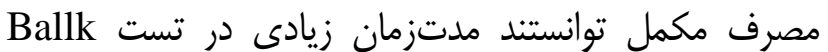
فعاليت كنند. در راستاى نتايج ما، فياضى و همكاران با بررسى ملى دمان

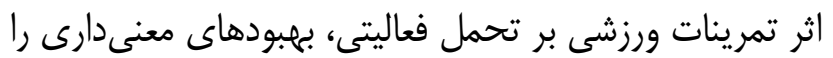

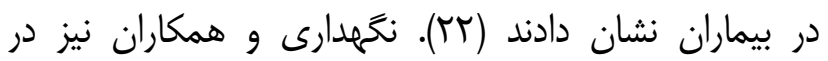

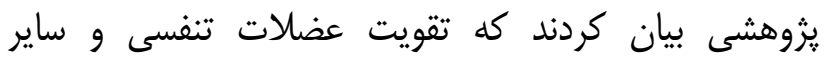

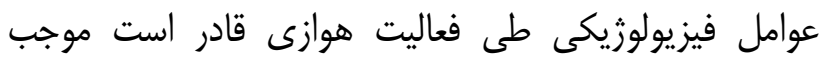

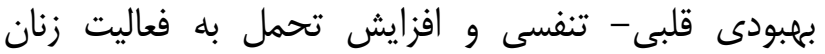

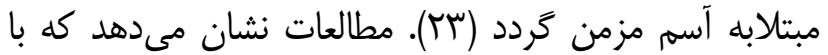
انجام فعاليت ورزشى منظم سبك همراه با افزايش تحمل به

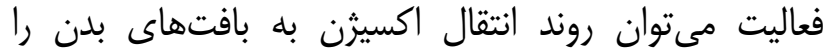
تسهيل كرد. افزايش در تحمل به فعاليت، روند انتقال اكسيثن

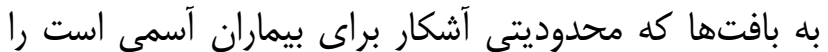

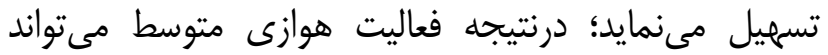

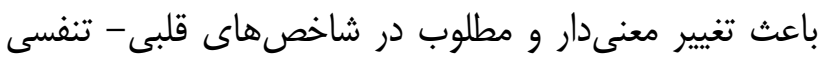

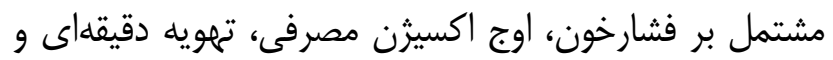

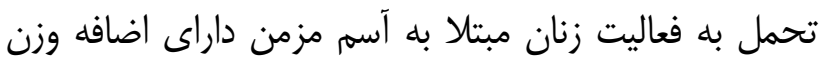

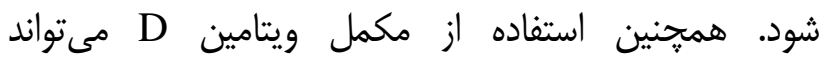

1- Moghaddasi B, Moghadasi Z, Taheri Nasab P. Effects of physical exercise on pulmonary function and clinical manifestations by asthmatic patients. J Arak Uni Med Sci. 2010; 13(2): 134-40. [Persian]

2- Kajbaf TZ, Asar S, Alipoor MR. Relationship between obesity and asthma symptoms among children in Ahvaz, Iran: a cross sectional study. Ital J Pediatr. 2011; 37: 1. doi: 10.1186/1824-7288-37-1.

3- Albers FC, Müllerová H, Gunsoy NB, Shin JY, Nelsen LM, Bradford ES, et al. Biologic treatment eligibility for realworld patients with severe asthma: the IDEAL study. J Asthma. 2018; 55(2): 152-60. doi: 10.1080/02770903.2017.1322611.

4- Hernandez CJ. Effects of Exercise in Pulmonary Rehabilitation on a Patient with Uncontrolled Symptomatic Asthma [Dissertation]. East Eisenhower: Florida Gulf Coast University, ProQuest LLC; 2019. 
5- Cilekar S, Tülek B, Kanat F, Süerdem M, Levendoglu F, Taşpınar IT. Effect of low-intensity pulmonary rehabilitation program on quality of life and pulmonary functions in patients with stable chronic obstructive pulmonary disease. Eurasian J Pulmonol. 2019; 21(1): 14-21. DOI: 10.4103/ejop.ejop_16_19

6- Asle Mohammadizadeh M, Ghanbarzadeh M, Habibi A, Nikbakht M, Taghavi Orveh A. The influence of two type's aerobic exercise in two different conditions on the thermal resistance of the airways resistance and bronchospasm athlete male students in Ahwaz City. Iran South Med J. 2014; 16(6): 447-58. [Persian]

7- Nogueira-Ferreira R, Moreira-Goncalves D, Santos M, Trindade F, Ferreira R, Henriques-Coelho T. Mechanisms underlying the impact of exercise training in pulmonary arterial hypertension. Respir Med. 2018; 134: 70-8. doi: 10.1016/j.rmed.2017.11.022.

8- Osho O, Akinbo S, Osinubi AA, Olawale OA. Effect of progressive aerobic and resistance exercises on the pulmonary functions of individuals with type 2 diabetes in Nigeria. Int J Endocrinol Metab. 2012; 10(1): 411-7. DOI: 10.5812/ijem.3333

9- Paul G, Brehm JM, Alcorn JF, Holguín F, Aujla SJ, Celedón JC. Vitamin D and asthma. Am J Respir Crit Care Med. 2012; 185(2): 124-32. doi: 10.1164/rccm.201108-1502CI.

10- Gomes TL, Fernandes RC, Vieira LL, Schincaglia RM, Mota JF, Nóbrega MS, et al. Low vitamin D at ICU admission is associated with cancer, infections, acute respiratory insufficiency, and liver failure. Nutrition. 2019; 60: 235-40. doi: 10.1016/j.nut.2018.10.018.

11- Gold DR, Litonjua AA, Carey VJ, Manson JE, Buring JE, Lee I-M, et al. Lung VITAL: Rationale, design, and baseline characteristics of an ancillary study evaluating the effects of vitamin D and/or marine omega-3 fatty acid supplements on acute exacerbations of chronic respiratory disease, asthma control, pneumonia and lung function in adults. Contemp Clin Trials. 2016; 47: 185-95. doi: 10.1016/j.cct.2016.01.003.

12- Moradgholi E, Jafari M, Fathei M. The effect of high-intensity interval training on E-selectin and P-selectin in obese women. Iran J Endocrinol Metab. 2016; 18(4): 279-86. [Persian]

13- Gupta A, Bush A, Hawrylowicz C, Saglani S. Vitamin D and asthma in children. Paediatr Respir Rev. 2012; 13(4): 236-43; quiz 243. doi: 10.1016/j.prrv.2011.07.003.

14- Szentpetery SE, Han YY, Brehm JM, Acosta-Pérez E, Forno E, Boutaoui N, et al. Vitamin D insufficiency, plasma cytokines, and severe asthma exacerbations in school-aged children. J Allergy Clin Immunol Pract. 2018; 6(1): $289-91$. e2.

15- Razavi Majd Z, Nazarali P, Hanachi P, Kordi MR. Effect of a course of aerobic exercise and consumption of vitamin D supplementation on respiratory indicators in patients with asthma. Majallah-i Dānishgāh-i Ulūm-i Pizishkī-i Qum, Qom Univ Med Sci J. 2013; 6(4): 74-80. [Persian]

16- Ogan D, Pritchett K. Vitamin D and the athlete: risks, recommendations, and benefits. Nutrients. 2013; 5(6): 185668.

17- Khosravi M, Tayebi SM, Ghorban-Nezhad N. Effects of eight weeks circuit resistance training on pulmonary function of inactive women. Ann Appl Sport Sci. 2013; 1(2): 11-8.

18- Barari A, Kazemi M, Abdi A. Training and Consumption of Glechoma Extracts on Selection of Spiro Metric Indices in Men with Respiratory Diseases. Razi J Med Sci. 2016; 23(146): 17-25. [Persian]

19- Odinets T, Briskin Y, Pityn M. Effect of individualized physical rehabilitation programs on respiratory function in women with post-mastectomy syndrome. Physiother Theory Pract. 2019 May;35(5):419-426. doi: 10.1080/09593985.2018.1444117.

20- Decramer M, Rutten-van Mölken M, Dekhuijzen PR, Troosters T, van Herwaarden C, Pellegrino R, et al. Effects of $\mathrm{N}$-acetylcysteine on outcomes in chronic obstructive pulmonary disease (Bronchitis Randomized on NAC Cost-Utility Study, BRONCUS): a randomised placebo-controlled trial. Lancet. 2005; 365(9470): 1552-60.

21- Akın Y, Ucar M, Yucel S. Actual Medical Management of Stone Diseases in Pediatric Population. Kuwait Medical Journal. 2014;46(3):196-206. 
22- Fayazi B, Parnow A, Ahsan B. Effect Aerobic Exercises on fatigue and quality of life in Women with Multiple Sclerosis. Journal of Holistic Nursing and Midwifery. 2016; 26(1): 30-40.

23- Negahdari S, Ghanbarzadeh M, Nikbakht M, Tavakol H. The Effect Of 8-Week Aerobic Training On Some Indices Of Cardiorespiratory And Exercise Tolerance In Overweight Women's With Chronic Asthma. Jundishapur Sci Med J. 2017; 16(3): 279-91. [Persian] 